\title{
FORMAL GROUPS OVER DISCRETE RINGS
}

\author{
BY ROBERT A. MORRIS AND BODO PAREIGIS ${ }^{1}$ \\ Communicated by Alex Rosenberg, September 8, 1972
}

In this note we develop a theory of formal schemes and groups over arbitrary commutative rings which coincides with that of [5] if the base ring is a field, and generalizes that of [2].

We assume always our base ring is discrete and treat a formal scheme (resp. group) $G$, with two principal tools: A topology on the affine algebra $\mathcal{O}(G)$ allows us to form its continuous linear dual $B(G)$, the coalgebra (resp. bialgebra) of distributions; a topology on $C^{*}$, the full linear dual of an arbitrary coalgebra $C$, allows recovery of the functor when given the distributions. In this way $B$ establishes an equivalence between our category of formal groups and a suitable Hopf algebra category. We discuss the Verschiebung and Frobenius maps, thus illuminating some of the literature on divided powers in Hopf algebras. The results presented here will be used elsewhere for the study of curves on a formal scheme $G$, or equivalently, of sequences of divided powers in $B(G)$. Detailed proofs will appear elsewhere.

Throughout $k$ is a commutative ring, algebra means commutative $k$-algebra, $k$-Alg is the category of commutative $k$-algebras, $\otimes$ means $\otimes_{k}$, etc. Func $=$ Func $(k-A l g$, Sets) is the category of set valued functors on $k$-Alg. Much terminology is standard and is collected, for example, in [4].

1. The affine algebra of a functor. Denote by $\mathcal{O}$ the underlying set functor on $k$-Alg. For any $G$ in Func, the $\operatorname{set} \mathcal{O}(G)=$ Func $(G, \mathcal{O})$ of natural transformations from $G$ to $\mathcal{O}$ is an algebra via pointwise operations, functorially in $G$. For each $A$ in $k$-Alg and each $x$ in $G(A)$, we denote by $\chi_{x, A}$ the algebra homomorphism $\mathcal{O}(G) \rightarrow A$ given by $\chi_{x, A}(f)=f(A)(x)$. Topologize $\mathcal{O}(G)$ with the coarsest topology making each of these maps continuous. If $k-\mathrm{Alg}_{c}$ denotes the category of topological $k$-algebras with continuous algebra homomorphisms, then $\mathcal{O}:$ Func $\rightarrow k-\mathrm{Alg}_{c}$ is a functor. $\mathcal{O}(G)$ is the affine algebra of $G$.

Let $\mathscr{A}$ be a topological algebra. Write $\mathscr{A}: k$-Alg $\rightarrow$ Sets for the functor $\mathscr{A}(K)=k-\mathrm{Alg}_{c}(\mathscr{A}, K)$, regarding $K$ with the discrete topology. Then ( ) $: k-\mathrm{Alg}_{c} \rightarrow$ Func is a functor which is adjoint on the right to $\mathcal{O}$, that is Func $(G, \mathscr{A})=k-\operatorname{Alg}_{c}(\mathscr{A}, \mathcal{O}(G))$. If we view $k$-Alg as a full subcategory of

AMS (MOS) subject classifications (1970). Primary 14L05.

${ }^{1}$ This research was partially supported by National Science Foundation Grants GU 3171 and PO 34003 while the second author was a visitor at SUNY at Albany. 
$k-\mathrm{Alg}_{c}$, then the functors $\mathscr{A}$ are restrictions of representable functors. Our formal schemes will have the property that the natural adjunction map $G \rightarrow \mathcal{O}(G)$ is an isomorphism (cf. [1, p. 101]).

We consider functors $G$ in Func equipped with a set of pairs $\left\{\left(D, p_{D}\right) \mid D\right.$ in $k$-Alg, $p_{D}$ in $\left.G(D)\right\}$ satisfying one or more of the axioms below. Associated with this collection will be a diagram $\mathscr{D}$ consisting of the $D^{\prime}$ 's and $\mathscr{D}\left(D, D^{\prime}\right)=$ $\left\{\alpha\right.$ in $\left.k-\operatorname{Alg}\left(D, D^{\prime}\right) \mid G(\alpha)\left(p_{D}\right)=p_{D^{\prime}}\right\}$ :

1.1 Each $\chi_{p_{D}, D}: \mathcal{O}(G) \rightarrow D$ is surjective.

1.2 Given any $A$ in $k$-Alg and $x$ in $G(A)$, there is a $D$ and an algebra map $y: D \rightarrow A$ such that $G(y)\left(p_{D}\right)=x$ (cf. $[\mathbf{1}$, p. 101]).

$1.3 \mathscr{D}$ is filtered.

1.4 Each $D$ is finitely generated and projective as a $k$-module.

If 1.1-1.2 hold we call the $p_{D}$ generic points of $G$. In this case the $y$ in 1.2 is necessarily unique, each $\mathscr{D}\left(D, D^{\prime}\right)$ has a single element and it is surjective.

1.5 Definition. The category Func gf $_{f}$ of formal schemes is the full subcategory of Func consisting of functors satisfying 1.1-1.3. The category $k$-Alg ${ }_{g f}$ of $g$ f-algebras is the full subcategory of $k$-Alg ${ }_{c}$ consisting of algebras $\mathscr{A}=\varliminf_{\mathrm{lim}} \mathscr{D}$ such that $\mathscr{D}=\{D, \mathscr{A} \rightarrow D\}$ satisfies $1.1-1.3$ (with respect to $\mathscr{A})$.

A $g f$-algebra is thus the filtered limit of algebras, with each projection surjective and with the property that any continuous map from the limit to a discrete algebra factors through one of these projections.

The adjoint functors $\mathcal{O}$ and ()$^{\circ}$ in fact provide an antiequivalence between $\mathrm{Func}_{\mathrm{g} f}$ and $k-\mathrm{Alg}_{g f}$. The latter category has finite coproducts, namely the complete tensor product $\mathscr{A} \hat{\otimes} \mathscr{B}=\underline{\lim }(\mathscr{D} \otimes \mathscr{E})$, where $\mathscr{A}=\varliminf_{\longleftarrow} \mathscr{D}$ and $\mathscr{B}=\lim _{\leftarrow} \mathscr{E}$.

2. Algebras and coalgebras. For any $\mathscr{A}$ in $k-\mathrm{Alg}_{c}$, let $\mathscr{A}^{\circ}$ denote the continuous linear dual $\operatorname{Hom}_{c}(\mathscr{A}, k)$. If $\mathscr{A}$ and $\mathscr{B}$ are $g f$-algebras which also satisfy 1.4 then $(\mathscr{A} \hat{\otimes} \mathscr{B})^{\circ}=\mathscr{A}^{\circ} \otimes \mathscr{B}^{\circ}$ as $k$-modules. For any such algebra $\mathscr{A}$ this allows a natural cocommutative $k$-coalgebra structure on $\mathscr{A}^{\circ}$. To pass back to the algebra category we topologize the full linear dual $C^{*}$ of an arbitrary cocommutative coalgebra $C$ with the finite annihilator topology, which has as base of open neighborhoods of zero those subgroups which are the annihilators of finite subsets of $C$. This makes $C^{*}$ a topological algebra even though these need not be ideals.

2.1 Definitions. The category $k-\mathrm{Alg}_{p f}$ is the full subcategory of $k-\mathrm{Alg}_{g f}$ consisting of algebras whose diagrams also satisfy 1.4. The category of flat formal schemes is the full subcategory Func $p_{f}$ of Func ${ }_{g f}$ consisting of formal schemes whose affine algebra is in $k$-Alg $\operatorname{sif}_{p} . k$-Coalg ${ }_{p f}$ is the full subcategory of the category of cocommutative coalgebras consisting of those coalgebras $C=\lim _{\longrightarrow} \mathscr{C}$ which are the colimit of a filtered diagram of 
coalgebras, each of which is finitely generated and projective as a $k$ module and with each natural map into the colimit split as $k$-module map.

The definition of $p f$-coalgebras forces sufficient flatness conditions that we may regard the elements $C_{\alpha}$ of $\mathscr{C}$ as subcoalgebras, despite the difficulty of defining the notion of subcoalgebras over arbitrary rings. For such a $\mathscr{C}$, the finite annihilator topology has a base of open neighborhoods of zero consisting of ideals, namely the annihilators of the $C_{\alpha}$.

()$^{\circ}$ and ()$^{*}$ define an antiequivalence between $k-\mathrm{Alg}_{p f}$ and $k$-Coalg (cf. [7, Chapter VI]. Note that over a field every coalgebra lies in $k$ Coalg $_{p f}$ ). If $\mathscr{A}$ and $\mathscr{B}$ are in $k-\mathrm{Alg}_{p f}$ then so is their coproduct $\mathscr{A} \hat{\otimes} \mathscr{B}$. This shows that the full embedding of Func $p f$ into Func $g f$ and also into Func preserves products.

2.2 Definition AND THEOREM. If $G$ is a flat formal scheme the coalgebra of distributions of $G$ is $B(G)=(\mathcal{O}(G))^{\circ}$. B is an equivalence of categories between Func Ff $_{p}$ and $k$-Coalg $p$.

A formal group (resp. flat formal group) is a group in $\mathrm{Func}_{g f}$ (resp. Func $_{p f}$ ). Either condition holds if and only if the formal scheme is a group valued functor.

If $G$ is a flat formal group, the group law induces a natural topological coalgebra structure $\mathcal{O}(G) \rightarrow \mathcal{O}(G) \hat{\otimes} \mathcal{O}(G)$ and in turn an algebra structure (and hence Hopf-algebra structure) on $B(G)$, which we call the bialgebra of $G$. This is the Hopf algebraic tool by which we elsewhere will describe a Dieudonné module theory for our flat formal groups via the curve groups of Cartier [3]. These curves can be identified with sequences of divided powers in $B(G)$.

2.3 THEOREM. $B$ defines an equivalence of the category of flat formal groups over $k$ with the category of cocommutative $k$-Hopf algebras whose coalgebra lies in $k$-Coalg ${ }_{p j}$.

3. Base extension. Let $K$ be a $k$-algebra. Then the restriction to $K$ algebras defines the base extension functor

$$
\text { Func ( } k \text {-Alg, Sets) } \ni G \mapsto G_{K} \in \text { Func ( } K \text {-Alg, Sets). }
$$

Base extension behaves reasonably well on flat formal schemes. If $G$ is a flat formal scheme over $k$ then $G_{K}$ is a flat formal scheme over $K$ and $\mathcal{O}(G)=\mathcal{O}(G) \hat{\otimes} K$ and $B\left(G_{K}\right)=B(G) \otimes K$. Since restriction of scalars need not take $K-\mathrm{Alg}_{p f}$ to $k-\mathrm{Alg}_{p f}$ it is not an adjoint to ( $)_{K}$ as happens for example for group schemes. Nevertheless, ()$_{K}$ preserves products on Func $_{p f}$ and hence flat formal groups. The usual formulation of Cartier duality holds for flat formal groups:

3.1 THEOREM. Let $G$ be a commutative flat formal group over $k$ and $K$ be any commutative k-algebra. Then $\operatorname{Hom}_{K-\mathrm{Gr}}\left(G_{K}, G_{m K}\right)=k$-Alg $(B(G), K)$ 
naturally in $G$ and $K$. Further, $\operatorname{Hom}_{K^{-G r}}\left(\operatorname{Hom}_{-}-G_{-}\left(G_{-}, G_{m}\right)_{K}, G_{m K}\right)=G(K)$.

Here $\mathrm{Hom}_{K-\mathrm{Gr}}$ denotes the homomorphisms in the category of groups in Func $_{g f}(K-\mathrm{Alg}$, Sets).

4. Frobenius and Verschiebung. Assume $\operatorname{char}(k)=p \neq 0$ is prime. Let $A$ be a $k$-algebra and denote by $A^{1 / p}$ the $k$-algebra whose ring is $A$ with $k$-algebra structure $k \stackrel{p}{\rightarrow} k \rightarrow A$ where $p(\beta)=\beta^{p}$. ( $)^{1 / p}$ is a functor from $k$-Alg to $k$-Alg and there is a natural transformation $F: \operatorname{Id}_{k-\mathrm{Alg}} \rightarrow()^{1 / p}$ called the Frobenius map and given by $F(A)(a)=a^{p}[4$, p. 270].

Now $p: k \rightarrow k^{1 / p}$ is a ring map making $k^{1 / p}$ into a $k$-algebra. Note that $k^{1 / p}=k$ as rings, so there is a category isomorphism ( $)^{\prime}$ from $k^{1 / p}$-Alg (resp. $k^{1 / p}$-Coalg) to $k$-Alg (resp. $k$-Coalg). For $\mathscr{A}$ in $k$-Alg $\lg _{g}$ let $\mathscr{A}^{(p)}$ denote $\left(A \hat{\otimes} k^{1 / p}\right)^{\prime}$ in $k-\mathrm{Alg}_{g f}$. Then $\left(\mathscr{A}^{(p)}\right)^{\cdot}(B)=\mathscr{A}^{\cdot}\left(B^{1 / p}\right)$ and there is a natural transformation $\mathscr{F}:()^{(p)} \rightarrow \mathrm{Id}_{k-\mathrm{Alg}_{g f}}$ such that

$$
\mathscr{F} \cdot(B): \mathscr{A} \cdot(B) \rightarrow\left(\mathscr{A}^{(p)}\right)^{\cdot}(B)
$$

is the map

$$
\mathscr{A}(B) \stackrel{\mathscr{A}(F)}{\longrightarrow} \mathscr{A} \cdot\left(B^{1 / p}\right)=\left(\mathscr{A}^{(p)}\right)^{\cdot}(B)
$$

$\mathscr{F}$ will also be called the Frobenius map.

We may define a functor ()$^{(p)}$ on $k$-Coalg by $C^{(p)}=\left(C \otimes k^{1 / p}\right)^{\prime}$. Then $C^{(p)}=\left(\left(C^{*}\right)^{(p)}\right)^{\circ}$ and there is a natural transformation $V: \operatorname{Id}_{k-\operatorname{Coalg}_{p f}} \rightarrow()^{(p)}$ obtained by dualizing $\mathscr{F}$. If $k$ is a field then these maps agree with the description of the Frobenius and Verschiebung given in [5], [6].

$V$ is expressed on $C$ in $k$-Coalg - by $V(C)(c)=\sum_{i} c_{i} \otimes \beta_{i}$, where $\sum c_{i} \otimes \beta_{i}$ is uniquely determined by the property that $\left(c, a^{p}\right)=\sum\left(c_{i}, a\right)^{p} \beta_{i}$ for any $a$ in $C^{*}$. This observation allows one to prove that $\mathscr{F}$ and $V$ are functorially Hopf algebra maps, or, more precisely, that $\mathscr{F}$ and $V$ are natural transformations of cogroups and groups resp., when restricted to the cogroups and groups resp. in $k-\mathrm{Alg}_{p f}$ and $k$-Coalg resp.

\section{BIBLIOGRAPHY}

1. P. Cartier, Groupes algébriques et groupes formels, Colloq. Théorie Groupes Algébriques (Bruxelles, 1962), Librairie Universitaire, Louvain; Gauthier-Villars, Paris, 1962, pp. 87-111. MR 26 \#6172.

2. - Groupes formels associés aux anneaux de Witt généralisés, C. R. Acad. Sci. Paris Sér. A-B 265 (1967), A49-A52. MR 36 \# 1448.

3. - Modules associé à un groupe formel commutatif. Courbes typiques, C. R. Acad. Sci. Paris Sér. A-B 265 (1967), A129-A132. MR 36 \# 1449.

4. M. Demazure and P. Gabriel, Groupes algébriques. Tome 1, North-Holland, Amsterdam, 1970.

5. P. Gabriel, Étude infinitésimale des schemas en groupes. Groupes formels, Exposé VIIB, Schémas en Groupes 1 (SGA 3) 1962/64, Lecture Notes in Math., vol. 151, Springer-Verlag, Berlin, 1970.

6. R. Heyneman and M. E. Sweedler, Affine Hopf algebras. II, J. Algebra 16 (1970), 
271-297. MR 42 \# 342.

7. M. E. Sweedler, Hopf algebras, Math. Lecture Notes Series, Benjamin, New York, 1969. MR 40 \# 5705.

Department of Mathematics, State University of New York at Albany, Albany, NEW YORK 12203

Mathematisches Institut, Universität MÜNChEN, MÜNChEN, Federal RePUblic OF GERMANY 